\title{
Decision support system for refinery site selection
}

\author{
Kamran Jamali Firouzabadi ${ }^{\mathrm{a}}$ and Mina Golshenas $\operatorname{Rad}^{\mathrm{b} *}$
}

${ }^{a}$ Faculty Member, Department of Industrial Engineering, Firouzkooh Branch, Islamic Azad University, Firouzkooh, Iran ${ }^{b}$ Masters student, Department of Industrial Engineering, Firouzkooh Branch, Islamic Azad University, Firouzkooh, Iran

\section{H R O N I C L E}

Article history:

Received December 10, 2013

Received in revised format

25 June 2014

Accepted June 262014

Available online

July 32014

Keywords:

Mathematical Model

Site Selection

Analytic Hierarchy Process

Multi-objective Model

Decision Support System

\section{A B S T R A C T}

Considering the importance and extensive range of decision-making, scientists from various fields have had many discussions on this issue. Various models have been proposed to facilitate decision-making and have had much utilization. In many site selection problems, multiple objectives must be obtained, simultaneously. This study uses a mathematical model to select a suitable location for the refinery in the multi attribute environment. The proposed model uses a large amount of qualitative and quantitative information in the frame of multi objective functions for the first time in the refinery site selection and is flexible enough to use decision makers' opinions in order to achieve goals. For this reason, after a brief overview of the selected area characteristics, using analytic hierarchy process (AHP) for weighting the criteria, a mathematical operation research model is proposed to determine the best alternatives.

(C) 2014 Growing Science Ltd. All rights reserved.

\section{Introduction}

The purpose of this paper is to explain the activities of selecting the location of a refinery site. We discuss not only the characteristic of each alternative in different aspects such as construction cost, passive defense, etc., but also we consider some other important elements of site selection such as surrounding seismic faults, evaluation of criteria regarding technical and economical optimization in order to meet decision maker's perspective and responding to the demands. The process of decisionmaking consists of two phases: the first phase, after criteria selection, weights each criterion using Analytic hierarchy process (AHP) method. During the second states of the mathematical model, objective functions are converted into single objective by assigning weights. In recent years, there are numerous site selection optimization models. Zaghian and Shahanaghy (2009) integrated AHP method and VIKOR in order to select the best site for a crude oil refinery. Karbasian and Abedi (2011) used a multi objective non-linear model considering passive defense principles. Yang and Jones (2007) proposed a method based on a combination of a fuzzy multi-objective programming and a genetic algorithm. The original fuzzy multiple objectives were converted into a single unified 'minmax' goal, which makes it easy to apply a genetic algorithm for the problem solving.

* Corresponding author Tel : +989123542334, +982128765525

E-mail address: m.golshenas@gmail.com (M. Golshenas Rad)

(C) 2014 Growing Science Ltd. All rights reserved.

doi: $10.5267 /$ j.uscm.2014.7.002 
Akbari and Rajabi (2008) integrated GIS and fuzzy multi criteria decision analysis (FMCDA) to solve the landfill site selection problem and to develop a ranking of the potential landfill areas based on a variety of criteria. Zhou and Li (2013) proposed a multi-objective goal-programming model, taking both service quality, setup costs, and operating costs into consideration in the uncertain environment.

\section{The proposed model}

\subsection{Assumptions of the model}

- The stage of feasibility study has fulfilled for every alternative before site selection considering required area for construction.

- Surrounding faults means faults within a radius of 150-kilometer of each alternative.

- Surrounding inhabited areas means inhabited areas within the radius of 12-kilometer of each alternative.

- Pipe material has been considered carbon steel.

- Sulfur has been considered granule, packed and rail transported connecting to the existing transrailways.

\subsection{Methodology}

\subsubsection{Phase 1: criteria selection and using AHP method}

In order to identify site selection effective factors, we used relevant expert's opinion in accordance with internationally acceptable standards/codes e.g. IPS (Iranian Petroleum Standards). One of the main factors affecting construction costs is length of pipelines; shorter refinery distance to wells is an advantage of each alternative from a technical and operational point of view. It is also very important to investigate the effect of earthquake ground motions potential and surrounding seismic faults based on relevant data and drawings. Environmental reviews are considered as well. Amount of pollutant dispersion is related to direction and velocity of prevailing wind. Refer to environmental and establishment industries criteria, production process and series of profiles approved by ministers, (Environmental and establishment industries criteria, 2000) minimum distance of some industries from some critical centers is described in Table 1 as follows,

\section{Table 1}

Minimum required distance from critical centers

\begin{tabular}{lll}
\hline Item & Critical center & Distance $(\mathrm{m})$ \\
\hline 1 & Inhabited areas & 1500 \\
2 & Educational centers & 100 \\
3 & Main roads & 150 \\
4 & International park & 1000 \\
5 & Protected areas /major rivers/canals & 300 \\
\hline
\end{tabular}

Risk assessment is required in every stage of the project. In this regard, probability estimation, measuring maximum concentration of pollutant must be carried out, maximum concentration of air pollutant must also be measured. In this level of the project, location and dimensions of equipment have not been determined. Thus, just the amount of air pollutant dispersion through flare and safe radius are measured by using PHAST software. (Safe radius: Within safe radius, concentration of toxic gas is less than quantity mentioned in standards to be safe) (e.g. less than $10 \mathrm{ppm}$ for H2S). (IPS-E-SF-860, 2010). Passive defense is a considered criterion. Vulnerability and threats of each type of attack e.g. high/low altitude aerial attack and risk factor are calculated.

\section{Weighting techniques}

Various methods can be used for weighting the criteria (e.g. decision maker's opinion or AHP based on pair wise comparison). In this paper, the second is used. (Momeni, 2010). 


\subsubsection{Phase 2: Mathematical Model}

This developed mathematical model is inspired by general site selection model (Bashiri, 2009).

$\operatorname{Min} \mathrm{Z}_{1}=\sum_{\mathrm{i} \in \mathrm{I}} \mathrm{C}_{\mathrm{i}} \mathrm{Y}_{\mathrm{i}}+\sum_{\mathrm{k} \in \mathrm{K}} \sum_{\mathrm{i} \in \mathrm{I}} \mathrm{CR}_{\mathrm{ki}} \mathrm{dr}_{\mathrm{ki}} \mathrm{Y}_{\mathrm{i}}+\sum_{\mathrm{k} \in \mathrm{K}} \sum_{\mathrm{i} \in \mathrm{I}} \mathrm{CV}_{\mathrm{ki}} \mathrm{X}_{\mathrm{ki}}$

This objective function seeks to minimize the costs of construction and sulfur transportation, where $\mathrm{C}_{\mathrm{i}}$ is construction cost of the gas refinery if it is established in alternative $i$ and defined as follows:

$\mathrm{C}_{\mathrm{i}}=\left(\mathrm{C}_{\mathrm{i}}^{1}+\mathrm{C}_{\mathrm{i}}^{2} \times \mathrm{d}_{\mathrm{IGAT} \mathrm{i}}+\mathrm{C}_{\mathrm{i}}^{3} \times \mathrm{d}_{\mathrm{IGAT} \mathrm{i}}+\mathrm{C}_{\mathrm{i}}^{4} \times \sum_{\mathrm{j} \in \mathrm{J}} \mathrm{d}_{\mathrm{ji}}+\mathrm{C}_{\mathrm{i}}^{5} \times \mathrm{d}_{\text {gathering } \mathrm{i}}+\mathrm{C}_{\mathrm{i}}^{6} \times \mathrm{d}_{\mathrm{hi}}+\mathrm{C}_{\mathrm{i}}^{7} \times \mathrm{dw}_{\mathrm{i}}+\mathrm{C}_{\mathrm{i}}^{8}\right)$

To calculate the cost of pipe, first we need to know their weight, wall thickness and size using formula below:

$\mathrm{W}_{\text {pipe }}=0.02466 \mathrm{t}\left(\mathrm{d}_{0}-\mathrm{t}\right)($ McAllister, 2013) $\mathrm{kg} / \mathrm{m}$

where $t$ is wall thickness $(\mathrm{mm}), \mathrm{d}_{0}$ is outside diameter, $\mathrm{mm}$, and $\mathrm{W}_{\text {total }}$ is total weight.

$\mathrm{W}_{\text {total }}=\mathrm{W}_{\text {pipe }} \times \mathrm{L}$

Where

$L$ is length of the pipe, $\mathrm{mm}$

Moreover, pipe wall thickness is as below:

$$
\mathrm{t}=\frac{\mathrm{Pd}_{0}}{2 \mathrm{SEFT}}+\mathrm{C} \cdot \mathrm{A} \text {. }
$$

where

$\mathrm{P}$, design pressure, $\mathrm{psi}$

$\mathrm{d}_{0}$, outside diameter, $\mathrm{mm}$

$S$, Specified Minimum Yield Strength, psi

$E$, Longitudinal Joint Factor

$F$, Basic Design Factor

$T$, Temperature De-rating Factor

C.A., Corrosion Allowance (ASME B31.8, 2010, ASME B36.10, 2004),

For calculation of power supply, regardless of substation and demand costs, which are identical in all alternatives, just the execution cost of transmission line is calculated.

$D L_{i} \times C E_{i}=C^{8}$

where

$\mathrm{DL}_{\mathrm{i}}$, length of execution line for each alternative

$\mathrm{CE}_{\mathrm{i}}$, execution cost per kilometer

$\mathrm{C}^{8}$, total execution cost 
$\operatorname{Max} \mathrm{Z}_{2}=\sum_{\mathrm{i} \in \mathrm{I}} \sum_{\mathrm{e} \in \mathrm{E}} \mathrm{d}_{\mathrm{ei}} \mathrm{Y}_{\mathrm{i}}$

Eq. (7) maximizes the sum of total surrounding fault distance of alternative.

$\operatorname{Max} \mathrm{Z}_{3}=\sum_{\mathrm{i} \in \mathrm{I}} \sum_{\mathrm{h} \in \mathrm{H}}\left(\mathrm{A}_{\mathrm{hi}}-\mathrm{I}_{\mathrm{i}}\right) \mathrm{Y}_{\mathrm{i}}$

Eq. (8) maximizes the sum of total difference of inhabited areas of alternative and its safe radius.

$\operatorname{Max} \mathrm{Z}_{4}=\sum_{\mathrm{i} \in \mathrm{I}} \sum_{\mathrm{l} \in \mathrm{l}}\left(\mathrm{L}_{\mathrm{li}}-\mathrm{D}\right) \mathrm{Y}_{\mathrm{i}}$

Eq. (9) maximizes sum of total deference of minimum required distance between the refinery and protected areas and protected areas.

$\max \mathrm{Z}_{5}=\sum_{\mathrm{i} \in \mathrm{I}} \mathrm{E}_{\mathrm{i}} \mathrm{Y}_{\mathrm{i}}$

$\min \mathrm{Z}_{6}=\sum_{\mathrm{i} \in \mathrm{I}} \sum_{\mathrm{m} \in \mathrm{M}} \mathrm{R}_{\mathrm{mi}} \mathrm{Y}_{\mathrm{i}}$

Alternatives are evaluated from the perspective of passive defense. This function minimizes total calculated risk,

$\mathrm{R}=\mathrm{EC} \times \mathrm{L} \times \mathrm{C}$

where

$E C$, impact for each stage of the attack

$L$, probability of occurrence

$C$, cost reduction index (FEMA 452, 2005)

Considering the following restrictions:

$\sum_{\mathrm{i} \in \mathrm{I}} \mathrm{Y}_{\mathrm{i}}=\mathrm{P}$

$\sum_{\mathrm{k} \in \mathrm{K}} \mathrm{X}_{\mathrm{ki}} \leq \mathrm{MY}_{\mathrm{i}} \quad \forall \mathrm{i} \in \mathrm{I}$

$\sum_{\mathrm{k} \in \mathrm{K}} \mathrm{X}_{\mathrm{ki}} \leq \mathrm{S} \quad \forall \mathrm{i} \in \mathrm{I}$

$\sum_{\mathrm{i} \in \mathrm{I}} \mathrm{C}_{\mathrm{i}} \mathrm{Y}_{\mathrm{i}}+\sum_{\mathrm{k} \in \mathrm{K}} \sum_{\mathrm{i} \in \mathrm{I}} \mathrm{CR}_{\mathrm{ki}} \mathrm{dr}_{\mathrm{ki}} \mathrm{Y}_{\mathrm{i}} \leq \mathrm{B}$

$\mathrm{Y}_{\mathrm{i}}=0$ or $1 \quad \forall \mathrm{i} \in \mathrm{I}$

$\mathrm{X}_{\mathrm{ki}} \geq 0 \quad \forall \mathrm{i} \in \mathrm{I} ; \forall \mathrm{k} \in \mathrm{K}$

Constraint (13) ensures that exactly $P$ alternatives are selected. Constraint (14) ensures no sulfur is transported to export port unless the alternative is selected. Constraint (15) shows capacity limitation of sulfur produced per day. Constraint (16) limits the amount of budget for establishment. Constraint (17) means that $Y_{i}$ is a binary variable, that is equal to 1 when alternative $i$ is selected and equal to 0 otherwise. Constraint (18) means that $\mathrm{X}_{\mathrm{ki}}$ is a positive variable.

2.3. Sets

I $\quad$ set of all potential alternatives (or sites)

$J \quad$ set of all wells

$K$ set of all ports to them the sulfur is transported for export

$L \quad$ set of all protected areas

$H \quad$ set of all surrounding inhabited areas 
$E \quad$ set of all surrounding fault

$M \quad$ set of all type of enemy's attack

\subsection{Parameters}

$P \quad$ Number of selected alternative

$B \quad$ total budget

$D \quad$ Minimum required distance of the protected area from the refinery

$S \quad$ Production capacity of sulfur per day

$E_{i} \quad$ Environmental score for each alternative

$\mathrm{A}_{\mathrm{hi}} \quad$ Distance of inhabited area $\mathrm{h}$ from alternative $\mathrm{i}$

$\mathrm{d}_{\mathrm{ei}} \quad$ Distance of fault e from alternative $\mathrm{i}$

$\mathrm{C}_{\mathrm{i}} \quad$ Fixed construction cost of the alternative

$\mathrm{C}^{1}{ }_{\mathrm{i}} \quad$ Earth working cost of each alternative (excavation and filling)

$\mathrm{C}^{2}{ }_{\mathrm{i}} \quad$ Cost of fuel transferring pipeline from IGAT (trans-pipeline) to the refinery per unit

$\mathrm{d}_{\text {IGAT I }}$ Trans-pipe line distance from the refinery if it's located in alternative $i$

$\mathrm{C}^{3}{ }_{\mathrm{i}} \quad$ Construction cost of gas pipeline from refinery to IGAT per unit

$\mathrm{C}^{4}{ }_{\mathrm{i}} \quad$ Construction cost of flow pipeline from well to gathering center alternative i per unit

$\mathrm{d}_{\mathrm{ji}} \quad$ Distance of well $\mathrm{j}$ from gathering center alternative $\mathrm{i}$.

$\mathrm{C}_{\mathrm{i}}^{5} \quad$ Construction cost of pipeline from gathering center to alternative i per unit.

$\mathrm{D}_{\text {gathering } \mathrm{i}} \quad$ Gathering center distance from the refinery established in alternative $\mathrm{i}$

$\mathrm{C}_{\mathrm{i}}^{6} \quad$ Construction cost of condensate pipeline to export port

$\mathrm{d}_{\text {hi }} \quad$ Distance of Established refinery in alternative $\mathrm{i}$ from export port

$\mathrm{C}_{\mathrm{i}}^{7} \quad$ Construction cost of road

$\mathrm{dw}_{\mathrm{i}} \quad$ Distance of the refinery established in alternative $\mathrm{i}$ from the existing road

$\mathrm{C}_{\mathrm{i}}^{8} \quad$ Power supply cost

$\mathrm{CR}_{\mathrm{ki}}$ Railway construction cost connecting export port $\mathrm{k}$ to alternative $\mathrm{i}$ via trans-railway

$\mathrm{dr}_{\mathrm{ki}} \quad$ Distance of alternative $\mathrm{i}$ from trans-railway ends to export port $\mathrm{k}$

$\mathrm{CV}_{\mathrm{ki}} \quad$ Variable cost of sulfur transportation from alternative $\mathrm{i}$ to export port $\mathrm{k}$

$\mathrm{L}_{\mathrm{li}} \quad$ Distance from protected area $\mathrm{l}$ from alternative $\mathrm{i}$

$\mathrm{I}_{\mathrm{i}} \quad$ Safe radius of alternative $\mathrm{i}$

$\mathrm{R}_{\mathrm{mi}} \quad$ Risk factor attack type $\mathrm{m}$ for alternative $\mathrm{i}$

\subsection{Decision variables}

$\mathrm{Y}_{\mathrm{i}} \quad=1$ if the refinery is established in alternative $\mathrm{i}$

or

$=0$ otherwise.

$\mathrm{X}_{\mathrm{ki}} \quad$ Quantity of sulfur transported to export port $\mathrm{k}$ from alternative $\mathrm{i}$

\section{Case study}

The proposed study of this paper assigns appropriate weights to convert the multi objective problem into a single objective problem so that we could solve the resulted problem using a simple linear programming package (Asgharpour, 2012).

\section{Table 2a}

The summary of information associated with alternatives

\begin{tabular}{ccccc}
\hline alternative & 1 & 2 & 3 & 4 \\
\hline $\mathrm{N}$ & 3013407.11 & 3021995.87 & 2993589.52 & 3021872.34 \\
$\mathrm{E}$ & 364884.32 & 401128.77 & 383415.54 & 441232.99 \\
Location & \multirow{2}{*}{$\begin{array}{c}60 \mathrm{~km} \text { from north west of } \\
\text { Bandar Abbas }\end{array}$} & $\begin{array}{c}30 \mathrm{~km} \text { from north } \\
\text { west of Bandar }\end{array}$ & $\begin{array}{c}45 \mathrm{~km} \text { from south } \\
\text { west of Bandar }\end{array}$ & $\begin{array}{c}17 \mathrm{~km} \text { from north } \\
\text { east of Bandar } \\
\end{array}$ \\
& & Abbas & Abbas & Abbas \\
\hline
\end{tabular}


Table 2b

The summary of information associated with alternatives

\begin{tabular}{cccc}
\hline S(ton) & D & B & P \\
\hline 1,000 & 300 & $1,800,000,000,000$ & 1 \\
\hline
\end{tabular}

Table 3 shows pair wise comparison of criteria. If more than one decision maker is needed based on a selection policy, GAHP can be used (Asgharpour, 2012). In addition, Table 4 shows the distances.

Table 3

Pairwise comparison of criteria

\begin{tabular}{|c|c|c|c|c|c|c|c|}
\hline & $\begin{array}{l}\text { Total } \\
\text { cost }\end{array}$ & $\begin{array}{l}\text { Sum of } \\
\text { fault } \\
\text { distance }\end{array}$ & $\begin{array}{c}\text { Distance of } \\
\text { inhabited } \\
\text { area }\end{array}$ & $\begin{array}{c}\text { Distance of } \\
\text { protected } \\
\text { area }\end{array}$ & $\begin{array}{l}\text { effecting on } \\
\text { environment } \\
\text { Score }\end{array}$ & $\begin{array}{l}\text { Passive } \\
\text { defense }\end{array}$ & $\begin{array}{c}\text { Relative } \\
\text { weight }\end{array}$ \\
\hline Total cost & 1 & 3 & 5 & 8 & 6 & 4 & 0.473 \\
\hline $\begin{array}{l}\text { Sum of fault } \\
\text { distance }\end{array}$ & 0.333 & 1 & 2 & 2 & 3 & 2 & 0.180 \\
\hline $\begin{array}{c}\text { Distance of } \\
\text { inhabited area }\end{array}$ & 0.2 & 0.5 & 1 & 2 & 2 & 1 & 0.109 \\
\hline $\begin{array}{c}\text { Distance of } \\
\text { protected area }\end{array}$ & 0.125 & 0.5 & 0.5 & 1 & 1 & 0.5 & 0.063 \\
\hline $\begin{array}{l}\text { effecting on } \\
\text { environment } \\
\text { Score }\end{array}$ & 0.167 & 0.333 & 0.5 & 1 & 1 & 0.5 & 0.062 \\
\hline Passive defense & 0.250 & 0.5 & 1 & 2 & 2 & 1 & 0.113 \\
\hline total & 2.075 & 5.833 & 10 & 14 & 17 & 9 & $\mathrm{IR}=0.006$ \\
\hline
\end{tabular}

\section{Table 4}

Distances

\begin{tabular}{lcccc}
\hline Distances from $(\mathrm{km})$ & \multicolumn{3}{c}{ Alternatives } \\
\hline Trans-railway & 43 & 5 & 24 & 18 \\
Main road & 1.7 & 2 & 1.5 & 1.5 \\
Protected area & 55 & 10 & 45 & 30 \\
\hline
\end{tabular}

\subsection{Calculations related to pollutant concentration}

The aim is to calculate the maximum concentration of $\mathrm{H}_{2} \mathrm{~S}$. Stack height and diameter are calculated (API521, 2007).

Stack height $(\mathrm{m}) \mathrm{H}:=92.94$

Stack Diameter (m) D: $=0.93$

Table 5

$\mathrm{H}_{2} \mathrm{~S}$ concentration

\begin{tabular}{|c|c|c|}
\hline alternative & Distance from the nearest inhabited area $(\mathrm{km})$ & Safe $\operatorname{radius}(\mathrm{km})$ \\
\hline 1 & 5 & 4 \\
\hline 2 & 4 & 5 \\
\hline 3 & 7 & 8 \\
\hline 4 & 5 & 7 \\
\hline
\end{tabular}

\section{Table 6}

Sum of difference between safe radius and inhabited areas

\begin{tabular}{ccccc}
\hline Alternative & 1 & 2 & 3 & 4 \\
\hline$\sum \mathrm{A}(\mathrm{H}, \mathrm{I})$ & 13,500 & 1,900 & $-1,000$ & 35,000 \\
\hline
\end{tabular}




\subsection{Pipe cost \& power supply calculation}

Table 7

Pipe cost calculation (Exclusive unit price of oil and gas refineries establishment, 2013)

\begin{tabular}{|c|c|c|c|c|c|}
\hline & & Alternative 1 & Alternative 2 & Alternative 3 & Alternative 4 \\
\hline fuel transferring pipeline & Length $(\mathrm{m}) \mathrm{d}_{\mathrm{IGAT} \mathrm{i}}$ & 500 & 2,500 & 24,000 & 8,000 \\
\hline from IGAT-4" & Thickness based on ASME B36.10 (mm) & \multicolumn{4}{|c|}{5,56} \\
\hline & Weight $(\mathrm{km} / \mathrm{m})$ & \multicolumn{4}{|c|}{14,91} \\
\hline & Cost per unit $\mathrm{C}_{\mathrm{i}}^{2} € *$ & \multicolumn{4}{|c|}{25} \\
\hline & Total cost $€$ & 191,000 & 955,000 & $9,168,000$ & $3,056,000$ \\
\hline gas pipeline to IGAT - & Length $(\mathrm{m}) \mathrm{d}_{\mathrm{IGAT} \mathrm{i}}$ & 500 & 2,500 & 24,000 & 8,000 \\
\hline \multirow[t]{4}{*}{$24 "$} & Thickness based on ASME B36.10 (mm) & \multicolumn{4}{|c|}{15,88} \\
\hline & Weight $(\mathrm{km} / \mathrm{m})$ & \multicolumn{4}{|c|}{232,67} \\
\hline & Cost per unit $\mathrm{C}_{\mathrm{i}}^{3} €$ & \multicolumn{4}{|c|}{382} \\
\hline & Total cost $€$ & 191,000 & 955,000 & $9,168,000$ & $3,056,000$ \\
\hline \multirow[t]{5}{*}{ flow pipeline $-6 "$} & Length $(\mathrm{m}) \sum \mathrm{d}_{\mathrm{ji}}$ & 55,000 & 32,000 & 32,000 & 32,000 \\
\hline & Thickness based on ASME B36.10 (mm) & \multicolumn{4}{|c|}{7,11} \\
\hline & Weight $(\mathrm{km} / \mathrm{m})$ & \multicolumn{4}{|c|}{28,26} \\
\hline & Cost per unit $C_{i}^{4} €$ & \multicolumn{4}{|c|}{47} \\
\hline & Total cost $€$ & $2,585,000$ & $1,504,000$ & $1,504,000$ & $1,504,000$ \\
\hline \multirow[t]{5}{*}{ gathering pipeline $-20 "$} & Length $(\mathrm{m}) \mathrm{d}_{\text {gathering } \mathrm{i}}$ & 500 & 35,000 & 25,000 & 78,000 \\
\hline & Thickness based on ASME B36.10 (mm) & \multicolumn{4}{|c|}{14,27} \\
\hline & Weight $(\mathrm{km} / \mathrm{m})$ & \multicolumn{4}{|c|}{173,75} \\
\hline & Cost per unit $C_{i}^{5} €$ & \multicolumn{4}{|c|}{285} \\
\hline & Total cost $€$ & 142,500 & $9,975,000$ & $7,125,000$ & $22,230,000$ \\
\hline \multirow[t]{5}{*}{ condensate pipeline $-4 "$} & Length $(\mathrm{m}) \sum \mathrm{d}_{\mathrm{ji}}$ & 45,000 & 36,000 & 24,000 & 48,000 \\
\hline & Thickness based on ASME B36.10 (mm) & \multicolumn{4}{|c|}{5,56} \\
\hline & Weight (km/m) & \multicolumn{4}{|c|}{14,91} \\
\hline & Cost per unit $\mathrm{C}_{\mathrm{i}}^{6} €$ & \multicolumn{4}{|c|}{25} \\
\hline & Total cost $€$ & $1,125,000$ & 900,000 & 600,000 & $1,120,000$ \\
\hline
\end{tabular}

* pipe cost : $1.64 €$ per $\mathrm{km} / 1 €=42,600$ Rials

Table 8

Power supply cost of alternatives

\begin{tabular}{ccc}
\hline Alternative & Length of execution line $(\mathrm{m})$ & Power supply cost(million rial) \\
\hline 1 & 35,000 & 140,000 \\
2 & 60,000 & 240,000 \\
3 & 15,000 & 60,000 \\
4 & 10,000 & 40,000 \\
\hline
\end{tabular}

\subsection{Volume of Earthwork and seismic faults}

Table 9

Volume of earthwork $\left(\mathrm{m}^{3}\right)$

\begin{tabular}{ccccc}
\hline Alternative & 1 & 2 & 3 & 4 \\
\hline Filling & 142,000 & 600,000 & $1,575,000$ & 250,000 \\
Excavation & $1,820,000$ & $5,850,000$ & 525,000 & $3,500,000$ \\
\hline Total cost(million rial) & 31,740 & 101,090 & 170,620 & 60,090
\end{tabular}

Table 10

Siesmic fault info of alternative 1

\begin{tabular}{ccc}
\hline Fault no. & Length $(\mathrm{km})$ & Distance of established area $(\mathrm{km})$ \\
\hline 1 & 27 & 25 \\
2 & 40 & 7 \\
3 & 140 & 20 \\
4 & 35 & 35 \\
\hline
\end{tabular}

Table 11

Siesmic fault info of alternative 2

\begin{tabular}{ccc}
\hline Fault no. & Length $(\mathrm{km})$ & Distance of established area $(\mathrm{km})$ \\
\hline 1 & 27 & 0 \\
2 & 3 & 1 \\
\hline
\end{tabular}


Table 12

Siesmic fault info of alternative 3

\begin{tabular}{ccc}
\hline Fault no. & Length $(\mathrm{km})$ & Distance of established area $(\mathrm{km})$ \\
\hline 1 & 150 & 10 \\
2 & 140 & 15 \\
3 & 15 & 20 \\
\hline
\end{tabular}

Table 13

Siesmic fault info of alternative 4

\begin{tabular}{ccc}
\hline Fault no. & Length $(\mathrm{km})$ & Distance of established area $(\mathrm{km})$ \\
\hline 1 & 20 & 20 \\
2 & 150 & 5 \\
3 & 15 & 17 \\
4 & 27 & 20 \\
\hline
\end{tabular}

\subsection{Environmental score}

Table 14

Final environmental score

\begin{tabular}{|c|c|c|c|c|}
\hline & \multicolumn{4}{|c|}{ Scores } \\
\hline & Water resources & Natural environment & Social environment/lands utilization & Total \\
\hline Alt 1 & 13 & 15 & 8 & 36 \\
\hline Alt 2 & 13 & 13 & 9 & 35 \\
\hline Alt 3 & 10 & 12 & 7 & 29 \\
\hline Alt 4 & 13 & 9 & 12 & 34 \\
\hline
\end{tabular}

\subsection{Passive defense data}

Table 15

Cost reduction index

\begin{tabular}{ccccccc}
\hline Attack Type & $\begin{array}{c}\text { High altitude } \\
\text { aerial }\end{array}$ & $\begin{array}{c}\text { Low altitude } \\
\text { aerial }\end{array}$ & Missile & Marine Artillery & Ground & partisan \\
\hline Index & $\% 72$ & $83 \%$ & $90 \%$ & $95 \%$ & $75 \%$ & $85 \%$ \\
\hline
\end{tabular}

Table 16

Attack type probability (\%)

\begin{tabular}{|c|c|c|c|c|c|c|c|}
\hline \multirow[b]{2}{*}{ alternative } & \multicolumn{7}{|c|}{ Attack Type } \\
\hline & $\begin{array}{c}\text { High altitude } \\
\text { aerial }\end{array}$ & $\begin{array}{c}\text { Low altitude } \\
\text { aerial }\end{array}$ & Missile & Marine Artillery & Ground & partisan & Mean \\
\hline 1 & 25 & 30 & 30 & 15 & 15 & 20 & 22.5 \\
\hline 2 & 45 & 10 & 25 & 15 & 15 & 20 & 21.67 \\
\hline 3 & 5 & 35 & 15 & 55 & 50 & 40 & 33.33 \\
\hline 4 & 25 & 25 & 30 & 15 & 20 & 20 & 22.5 \\
\hline
\end{tabular}

Table 17

Total calculated risk

$\begin{array}{ccccc}\text { Alternative } & 1 & 2 & 3 & 4 \\ \sum \mathrm{R}(\mathrm{M}, \mathrm{I}) & 0.1705 & 0.1525 & 0.2512 & 0.1690\end{array}$

\section{Table 18}

Impact index for each stage of the attack

\begin{tabular}{|c|c|c|c|c|c|}
\hline \multirow[b]{2}{*}{ Attack Type } & \multicolumn{3}{|c|}{ Attack Stage } & \multirow[b]{2}{*}{ Target } & \multirow[b]{2}{*}{ damage } \\
\hline & Identity & Presence & Recognition & & \\
\hline High Altitude Aerial & 10 & 5 & 10 & 25 & 50 \\
\hline Low Altitude Aerial & 10 & 10 & 10 & 20 & 50 \\
\hline Missile & 10 & 5 & 15 & 20 & 50 \\
\hline Marine Artillery & 20 & 15 & 15 & 25 & 25 \\
\hline Ground & 5 & 35 & 5 & - & 40 \\
\hline Partisan & 5 & 35 & - & 5 & 35 \\
\hline
\end{tabular}


Table 19

Software results

\begin{tabular}{ccc}
\hline Selected alternative & Optimal value of objective function & Alternative1 \\
\hline & Objective function 1 & $9.84226 \mathrm{E}+11$ \\
Objective function 2 & 87000.000 \\
Objective function 3 & 13500.000 \\
Objective function 4 & 54700.000 \\
Objective function 5 & 43.000 \\
Objective function 6 & 0.171 \\
\hline Alternative1 & Construction cost \\
\hline Alternative2 & $3.82226 \mathrm{E}+11$ \\
Alternative3 & $1.01988 \mathrm{E}+12$ \\
Alternative4 & $1.21577 \mathrm{E}+12$ \\
\hline Quantity of sulfur transported & $1.36633 \mathrm{E}+12$ \\
\hline
\end{tabular}

\section{Conclusion}

In this paper, a unique model was presented, which could select the refinery site in order to consider every important site selection criterion specifically the effect of construction cost, earthquake ground motions potential, surrounding seismic faults in accordance with internationally acceptable standards and also passive defense in a manner that the possibility of damages by the enemy being reduced. In this developed mathematical model, technical terms met decision maker's perspective with the help of GAMS software. The model has been demonstrated through the case study in this paper. The case has shown how effectively this model could be applied in the process of selecting an alternative for establishing a refinery site. However, this model can be used to select the appropriate location for any other sites e.g. manufacturing plants, etc.

\section{References}

Asgharpour, M. (2012). Multiple Criteria Decision Making. University of Tehran.

ASME (American Society of Mechanical Engineers) (2010). Gas Transmission and Distribution Piping Systems, B31.8, 54-58.

ASME (American Society of Mechanical Engineers) (2004). Welded and Seamless Wrought Steel Pipe, B36.10.M, 10-25.

Akbari, V., \& Rajabi, M. (2008). Landfill site selection by combining GIS and fuzzy multi criteria decision analysis, Case Study: Bandar Abbas, Iran. World Applied Sciences Journal 3(1), 39-47.

American Petroleum Institute, API521 (2007). 137-171.

Bashiri, M., (2009), Facilities Planning, Shahed university.

Exclusive unit price of oil and gas refineries establishment. (2013). Ministry of oil.

FEMA (Federal Emergency Management Agency), 452, Risk Assessment A How to Guide to Mitigate Potential Terrorist Attacks Against Buildings, (2005).

International Institute of Seismology and Earthquake Engineering, Faults atlas, (2012).

IPS-E-SF-860 (Iranian Petroleum Standards) Standard for Air Pollution Control, (2007), First Edition, Appendix G.

Iran Railroad Line Atlas, (2013).

Iran Road Atlas, (2010).

Iskandar, F., (2009) Iran wildlife, Academic publication center.

Karbasian, M., \& Abedi, S. (2011). A Multiple Objective Nonlinear Programming Model for Site Selection of the Facilities Based on the Passive Defense Principles. International Journal of Industrial Engineering and Production Research, 22(4), 243-250.

Land affair organization. (2004). Execution Instructions, Volume II. 
Mansoori, J. (2013). A Guide to Birds of Iran, Farzaneh books.

McAllister, E.W. (2013). Pipe line rules of thumb handbook: A manual of quick, accurate solution to every day pipeline engineering problems, $8^{\text {th }}$ ed., 109-113.

Monavari, M. (2007). Environmental Impact Assessment Guidelines, Farzaneh books.

Momeni, M. (2010), New Topics in Operations and Research, $1^{\text {st }}$ ed., Agah publication.

Protected Area Atlas. (2007). The Environmental Protection organization.

The Environmental Protection organization. (2004). Environmental Criteria and Standards Regulations.

The underground waters of Hormozgan province. (2012). The Institute for Planning and Agricultural Economics.

Yang, L., Jones, B. F., \& Yang, S. H. (2007). A fuzzy multi-objective programming for optimization of fire station locations through genetic algorithms. European Journal of Operational Research, 181(2), 903-915.

Zaghian, M., Shahanaghy, K. (2009). Integrating AHP and VIKOR methods to locate oil refinery, $6^{\text {th }}$ International Conference on Industrial Engineering, Tehran, Iran Institute of Industrial Engineering, Sharif University of Technology.

Zhou, J., \& Li, Zh. (2013). A multi-objective model for fire station location under uncertainty, Advances in information Sciences and Service Sciences(AISS), 5(6)

Ziaee, H. (2009). Field Guide to Mammals of Iran, Introduction to Wildlife Center. 